\title{
RIGHT INVERTIBLE MULTIPLICATION OPERATORS AND STABLE RATIONAL MATRIX SOLUTIONS TO AN ASSOCIATE BEZOUT EQUATION, II: DESCRIPTION OF ALL SOLUTIONS
}

\author{
A. E. Frazho, M. A. KaAshoek and A. C. M. Ran
}

\begin{abstract}
This paper presents a state space description of the set of all solutions to a rational corona type Bezout equation, starting from a stable state space representation of the given coefficient matrix. In other words, we describe the null space of an analytic Toeplitz operator with a rational symbol, in terms of the matrices occuring in a realization of that symbol, assuming the operator involved is right invertible. A state space version of the related Tolokonnikov lemma is also included.
\end{abstract}

Mathematics subject classification (2010): Primary 47B35, 39B42; Secondary 47A68, 93B28.

Keywords and phrases: Right invertible multiplication operator, Toeplitz operators, Bezout equation, stable rational matrix functions, state space representation, discrete algebraic Riccati equation, stabilizing solution.

\section{REFERENCES}

[1] L. CARLESON, Interpolation by bounded analytic functions and the corona problem, Ann. Math. 76 (1962), 547-559.

[2] A. E. FrAZHO AND W. BosRI, An operator perspective on signals and systems, OT 204, Birkhäuser Verlag, Basel, 2010.

[3] A. E. Frazho, M. A. KaAshoek, AND A. C. M. Ran, Right invertible multiplication operators and $H^{2}$ solutions to a rational Bezout equation, I. Least squares solution, Integral Equations and Operator Theory 70 (2011), 395-418.

[4] P. Fuhrmann, On the corona theorem and its applications to spectral problems in Hilbert space, Trans. Amer. Math. Soc. 132 (1968), 55-66.

[5] I. Gohberg, S. Goldberg, And M. A. KaAshoek, Classes of Linear Operators, Volume I, OT 63, Birkhäuser Verlag, Basel, 1993.

[6] I. Gohberg, P. Lancaster, And L. Rodman, Matrix polynomials, Academic Press, New York, 1982.

[7] T. KaIlath, Linear systems, Prentice-Hall, Inc., Englwoords Cliffs, N.J., USA, 1980.

[8] N. K. NiKOL'S KII, Treatise on the shift operator, Grundlehren 273, Springer Verlag, Berlin 1986.

[9] M. Rosenblum and J. Rovnyak, Hardy Classes and Operator Theory, Oxford University Press, New York, 1985.

[10] V. A. Tolokonnikov, Estimates in Carleson's corona theorem. Ideals of the algebra $H^{\infty}$, the problem of Szókefalvi-Nagy, Zap. Naučn. Sem. Leningrad. Otdel. Mat. Inst. Steklov. (LOMI) 113 (1981), 178-1981 (Russian).

[11] T. T. TREnt, An algorithm for the corona solutions on $H^{\infty}(D)$, Integral Equations and Operator Theory 59 (2007), 421-435. 